\title{
Multiple-Micronutrient Fortified Non-Dairy Beverage Interventions Reduce the Risk of Anemia and Iron Deficiency in School-Aged Children in Low-Middle Income Countries: A Systematic Review and Meta-Analysis ${ }^{(i-i v)}$
}

\section{Grant J. Aaron ${ }^{1, \dagger, *}$, Daphna K. Dror ${ }^{2, \dagger}$ and Zhenyu Yang ${ }^{3, \dagger}$}

1 Global Alliance for Improved Nutrition (GAIN), Rue de Vermont 37-39, Geneva CH-1202, Switzerland

2 United States Department of Agriculture (USDA), Western Human Nutrition Research Center (WHNRC), 430 W. Health Sciences Dr., Davis, CA 95616, USA; E-Mail: daphnadror@gmail.com

3 Key Laboratory of Trace Element Nutrition, Ministry of Health of China, National Institute for Nutrition and Health, Chinese Center for Disease Control (CCDC), No. 29 Nanwei Road, Xicheng District, Beijing 100050, China; E-Mail: yang.zhenyuid@gmail.com

$\dagger$ These authors contributed equally to this work.

* Author to whom correspondence should be addressed; E-Mail: gjaaron@gainhealth.org; Tel.: +41-22-749-1850; Fax: +41-22-749-1851.

Received: 26 February 2015 / Accepted: 5 May 2015 / Published: 21 May 2015

\begin{abstract}
Multiple-micronutrient (MMN) fortification of beverages may be an effective option to deliver micronutrients to vulnerable populations. The aim of the present systematic review and meta-analysis is to evaluate the nutritional impacts of MMN fortified beverages in the context of low-middle income countries. A systematic search of published literature yielded 1022 citations, of which 10 randomized controlled trials (nine in school-aged children and one in pregnant women) met inclusion criteria. Results of school-aged children were included in the meta-analysis. Compared to iso-caloric controls, children who received MMN fortified beverages for 8 weeks to 6 months showed significant improvements in hemoglobin $(+2.76 \mathrm{~g} / \mathrm{L}, 95 \%$ CI $[1.19,4.33], p=0.004$; 8 studies) and serum ferritin ( $+15.42 \mathrm{pmol} / \mathrm{L},[5.73,25.12], p=0.007 ; 8$ studies $)$; and reduced risk of anemia (RR 0.58 [0.29, 0.88], $p=0.005 ; 6$ studies), iron deficiency (RR 0.34 [0.21, 0.55], $p=0.002 ; 7$ studies), and iron deficiency anemia (RR 0.17 [0.06, 0.53], $p=0.02 ; 3$ studies). MMN fortified beverage interventions could have major programmatic implications for reducing the burden of anemia and iron deficiency in school-aged children
\end{abstract}


in low-middle income countries. Additional research is needed to investigate effects on other biochemical outcomes and population subgroups.

Keywords: fortification; multiple-micronutrients; beverages; children; low-income

\section{Introduction}

In resource poor settings, vulnerable population groups including children and women of reproductive age are prone to multiple micronutrient $(\mathrm{MMN})$ deficiencies primarily due to relatively high requirements, poor dietary quality [1] and/or increased susceptibility to infection [2,3]. For example, globally iron deficiency anemia affects $18 \%$ and $19 \%$ of children under-5 years and pregnant women respectively, while $35 \%$ of children under-5 years and $15 \%$ of pregnant women suffer from vitamin $\mathrm{A}$ deficiency. Iodine and zinc deficiencies are estimated to affect $29 \%$ and $17 \%$ of the world's population [4]. During pregnancy and early childhood, MMN deficiencies increase maternal, infant, and child morbidity and mortality and prevent surviving offspring from attaining full developmental capacity [4]. Interventions that deliver $\mathrm{MMN}$ to these populations are therefore warranted, both to enhance nutritional outcomes compared to single micronutrient interventions [1], and to increase cost-effectiveness of delivery [5].

The two most widely used strategies for delivering additional micronutrients are: (1) supplementation — defined as the provision of micronutrients in capsule, tablet, or syrup form; and (2) fortification - for the purposes of the current paper defined as micronutrients added to processed food vehicles, or at point-of-use (home fortification) [6,7]. The appropriateness, extent of use, and programmatic success of these approaches vary considerably by country and context, depending on the population subgroups targeted, the nature and severity of deficiencies, as well as the financial and technical resources to develop and sustain delivery [8]. Supplementation and fortification are generally considered complementary strategies, with the former most suitable in contexts where certain population sub-groups may not be reached, or when nutrient requirements may not be met by other intervention strategies. Fortification has several advantages in terms of consumer acceptance, relative ease of fortifying foods, reach and coverage, cost-effectiveness, and sustainability [6]. Numerous international organizations, governments, consensus meetings, and more recently the Scaling Up Nutrition (SUN) movement, have endorsed fortification as one of the key strategies to reduce MMN deficiencies in high-burden countries [9]. Most programmatic experience in this context to date has focused on large-scale fortification of staple foods targeted to the general population, and more recently to home (or point-of-use) fortification products targeted to infants and young children. MMN fortification of beverages, though not widely implemented, may be an effective option to cover gaps in other existing nutrition strategies. Some advantages of beverages as vehicles for fortification include high consumer acceptance, achieved via potential fortification of familiar beverages, and flexible delivery as a ready-to-consume (RTC) beverage products or as powder which can be reconstituted [10-12].

The aim of the present systematic review and meta-analysis is to review the nutritional impacts of non-dairy fortified beverages in the context of low-middle income countries. The primary focus of the review is on school aged children due to the paucity of studies in other population groups. Dairy 
beverages were excluded to limit potential confounding effects of other nutritional factors present in milk products in the absence of non-dairy MMN fortified beverage control groups.

\section{Methods}

\subsection{Search Strategy}

The systematic review was conducted in line with the guidelines for Preferred Reporting Items for Systematic Reviews and Meta-Analyses (PRISMA). A systematic search of published literature from 1 January 1966 through 28 February 2013 was undertaken, and subsequently updated on 15 February 2015, using the US national Library of medicine's MEDLINE/PubMed bibliographic search engine. Multiple PubMed searches were conducted using various combinations of Medical Subject Heading (MeSH) and Title/Abstract (TIAB) keywords (Table 1). A filter was used to select only studies conducted on humans; no filters were set for language restrictions. All co-authors independently screened unique article titles and abstracts using a standardized form to identify a short list of studies for further review. When opinions differed, discrepancies were resolved by co-author consensus or by two-thirds majority if consensus was not achieved. The same process as above was carried out to identify the subset of articles to include for qualitative review. For those articles included in the qualitative review, the following data were extracted: setting, study population, study design, duration, intervention formulation, placebo formulation, frequency of beverage administration, and outcomes assessed. For biochemical and hematological outcomes, all data except hemoglobin were converted to international system (SI) units.

Table 1. PubMed search strategy.

\begin{tabular}{|c|c|c|}
\hline Step & Search Strategy $1^{1}$ & Search Strategy $2^{1}$ \\
\hline 1 & "beverages" $[\mathrm{MeSH}]^{2} \mathrm{NOT}$ “milk" $[\mathrm{MeSH}]$ & Beverage $* 3[$ TIAB $]{ }^{4}$ NOT milk $*[$ TIAB $]$ \\
\hline 2 & "food, fortified" [MeSH] & Fortif* [TIAB] \\
\hline 3 & 1 AND 2 & 1 AND 2 \\
\hline 4 & "beverages" [MeSH] NOT “milk" [MeSH] & Beverage * [TIAB] NOT milk * [TIAB] \\
\hline 5 & "micronutrients" [MeSH] & $\begin{array}{c}\text { Micronutrient } *[\text { TIAB }] \text { OR vitamin * }[\text { TIAB }] \\
\text { or mineral } *[\text { TIAB] OR iron }[\text { TIAB] OR } \\
\text { vitamin A }[\text { TIAB] OR zinc }[\text { TIAB }]\end{array}$ \\
\hline 6 & 4 AND 5 & 4 AND 5 \\
\hline 7 & 3 OR 6 & 3 OR 6 \\
\hline
\end{tabular}

In order to identify any additional articles not found by the PubMed searches, the reference sections for full articles reviewed were searched, and subject matter experts were consulted. An external scientific advisory board consisting of two subject matter experts was consulted to ensure that no related articles were missed in our analyses. 


\subsection{Study Eligibility Criteria}

Studies considered for selection assessed the effects of non-dairy MMN fortified beverages (RTC or powders for reconstitution) on micronutrient status, anthropometry, morbidity, and/or functional outcomes. Only single or double blind randomized controlled trials (RCT) with treatment and placebo groups conducted in the context of low-middle income countries, on apparently healthy children and women of reproductive age were considered. No restrictions were made for the number of study arms, micronutrient formulations, or study duration.

\subsection{Statistical Analyses}

For continuous outcomes, pooled SDs of the change were calculated if the change from baseline to end line and their SDs were reported. Final sample sizes were used to calculate the pooled SD if the number of participants decreased during the course of the study. If the change was not reported, the difference in final value between the intervention and control group was calculated and the SD of the final values from each group was used to calculate pooled SD. If the SD was not reported, 95\%-CI of mean values were converted to SD; or medians with 25 th and 75 th percentiles were converted to mean and SD. For binary data, risk ratios and $95 \%$-CI were calculated. Differences were considered significant at $\alpha=0.05$.

For trials with more than two groups, the comparison group most similar to MMN-fortified beverage $v s$. iso-caloric control was selected for the analysis $[13,14]$. One trial involved a 12-month intervention with results reported as change from 0-6 and 6-12 months [15]. For comparability with other designs only results from the $0-6$ month period were included in the meta-analysis.

\subsection{Assessment of Heterogeneity, Sensitivity, and Risk of Bias}

A random-effects model was used to account for heterogeneity across studies, with $\mathrm{I}^{2}$ statistic calculated for each meta-analysis. Heterogeneity cutoffs were classified as: low $<25 \%$; moderate 25\%-75\%; and high $>75 \%$ [16]. Meta-regression analyses were performed to evaluate relationships between predetermined independent factors: micronutrient dose, intervention duration, baseline values, deworming, and the most commonly reported outcomes (hemoglobin, serum ferritin or weight). Study bias was assessed by publication bias, the method of randomization, type of blinding (single or double), the percentage of loss to follow-up (low vs. high) and subgroup analyses.

\section{Results}

\subsection{Search Results and Study Characteristics}

The PubMed literature searches yielded 1158 citations updated on 15 February 2015. After removing duplicates $(n=136)$, titles and abstracts of 1022 citations were screened. Of these citations, 996 studies were excluded for various reasons (Supplemental Figure S1). Full texts were screened for 26 studies, of which 9 studies were included in the initial qualitative review. The reference sections for full texts yielded no additional studies. One co-author found a relevant study that was not yet indexed by 
PubMed after the initial qualitative review [11], which was eligible and subsequently included in the systematic review.

The final set of 10 studies included a total of 4645 participants who had both baseline and end line measurements (Table 2). Studies were conducted in Bangladesh, Botswana, India, Nigeria, the Philippines, South Africa, and Tanzania. All studies were double-blind RCT; 9 trials were conducted in school children [10,11,13-15,17-20] and one trial was conducted in pregnant women [21]. The mean age range of children participating in the included studies was 5-18 years, and the mean age of women in the adult trial was 25 years. Eight of the trials had two study groups (MMN fortified beverage and iso-caloric control beverage), one trial also included a non-intervention control group, and one trial had four study groups including a MMN-fortified non-caloric beverage and an unfortified non-caloric control. End line sample sizes for the selected studies ranged from 89-989 (median 331; IQR 247-714), and study durations ranged from 2-12 months (median 4; IQR 2-6). Eight of the trials tested beverage powders, and two trials tested RTC beverages; various MMN formulations were tested (Supplemental Table S1). The selected trials evaluated the following outcomes: hemoglobin $(n=9)$; ferritin $(n=9)$; retinol $(n=6)$; zinc $(n=4)$; vitamin B12 $(n=3)$; folate $(n=3)$; vitamin $\mathrm{C}(n=2)$; riboflavin $(n=2)$; vitamin B6 $(n=1)$; thiamin $(n=1)$; niacin $(n=1)$; iodine $(n=1)$; anthropometry $(n=8)$; cognition $(n=3)$; and physical performance $(n=2)$.

\subsection{Methodological Quality}

Methodological quality was considered moderate for 7 of 10 studies following downgrades for inadequate randomization or allocation concealment, high loss to follow up, or poor generalizability. Quality of the remaining individual trials was considered low $(n=1)$ and high $(n=2)$ (Table 3). At the outcome level, overall quality of the evidence was determined to be moderate for most outcomes relating to iron status and anthropometry and low or very low for remaining outcomes due to inadequate number of data points and/or high heterogeneity (Table 4). 
Table 2. Study descriptions and main results for studies included in the review.

\begin{tabular}{|c|c|c|c|c|c|c|}
\hline Reference & Population & Intervention & Control & Duration & Outcomes & Main Results \\
\hline $\begin{array}{l}\text { Aaron et al., } \\
2011[17]\end{array}$ & $\begin{array}{l}\text { Country: Nigeria } \\
\text { Geography: rural } \\
\text { Population: M and F } 5-13 \text { years } \\
(n=566) \\
\text { Exclusion criteria: } \\
\mathrm{Hb}<70 \mathrm{~g} / \mathrm{L} \text {, signs of acute illness }\end{array}$ & $\begin{array}{l}\text { RTC beverage fortified } \\
\text { with: } 11 \text { vitamins, } \\
12 \text { minerals, and } \\
\text { bioflavonoids }\end{array}$ & $\begin{array}{l}\text { Iso-caloric non- } \\
\text { fortified beverage }\end{array}$ & 6 months & $\begin{array}{l}\mathrm{Hb} \text {, ferritin, retinol, zinc, } \\
\text { anthropometry, and } \\
\text { cognitive performance }\end{array}$ & $\begin{array}{l}\text { Change in } \mathrm{Hb} \text {, retinol and zinc } \\
\text { significantly greater in fortified } \\
\text { group. No significant difference } \\
\text { between groups in } \mathrm{Hb} \text {, ferritin, } \\
\text { anthropometry, or cognitive } \\
\text { performance. }\end{array}$ \\
\hline $\begin{array}{l}\text { Abrams et al., } \\
2003[18]\end{array}$ & $\begin{array}{l}\text { Country: Botswana } \\
\text { Geography: urban } \\
\text { Population: M and F } 5-11 \text { years } \\
(n=311) \\
\text { Exclusion criteria: } \mathrm{Hb} \leq 60 \mathrm{~g} / \mathrm{L}, \\
\text { weight } \leq 15 \mathrm{~kg} \text {, known chronic or acute } \\
\text { illness }\end{array}$ & $\begin{array}{l}\text { Beverage reconstituted } \\
\text { from powder fortified } \\
\text { with: } 8 \text { vitamins and } \\
4 \text { minerals }\end{array}$ & $\begin{array}{l}\text { Iso-caloric non- } \\
\text { fortified beverage } \\
\text { reconstituted from } \\
\text { powder }\end{array}$ & 8 weeks & $\begin{array}{l}\mathrm{Hb} \text {, ferritin, transferrin } \\
\text { receptors, retinol, } \\
\text { vitamin } \mathrm{B} 12 \text {, folate, } \\
\text { riboflavin, zinc, } \\
\text { anthropometry }\end{array}$ & $\begin{array}{l}\text { Change in } \mathrm{Hb} \text {, ferritin, folate and } \\
\text { riboflavin; final transferrin } \\
\text { receptors, zinc adequacy, and } \\
\text { anthropometry significantly } \\
\text { greater in fortified group. No } \\
\text { significant difference between } \\
\text { groups in retinol or vitamin B12. }\end{array}$ \\
\hline $\begin{array}{l}\text { Angeles-Agdeppa } \\
\text { et al. } 2011[10]\end{array}$ & $\begin{array}{l}\text { Country: Philippines } \\
\text { Geography: urban } \\
\text { Age: M and F } 6-9 \text { years } \\
(n=100) \\
\text { Exclusion criteria: } \\
\mathrm{Hb}>120 \mathrm{~g} / \mathrm{L} \text { or } \mathrm{Hb}<70 \mathrm{~g} / \mathrm{L}, \mathrm{WAZ}<-3) \text {, } \\
\text { acute illness }\end{array}$ & $\begin{array}{l}\text { RTC beverage fortified } \\
\text { with: } 2 \text { vitamins, } \\
2 \text { minerals, and lysine. }\end{array}$ & $\begin{array}{l}\text { Iso-caloric beverage } \\
\text { fortified only with } \\
\text { Vitamin C }\end{array}$ & 100 days & $\begin{array}{l}\mathrm{Hb} \text {, ferritin, zinc, } \\
\text { anthropometry, } \\
\text { adequacy of energy and } \\
\text { nutrient intake }\end{array}$ & $\begin{array}{l}\text { Change in } \mathrm{Hb} \text { and zinc } \\
\text { significantly greater in fortified } \\
\text { group. No significant difference in } \\
\text { ferritin or anthropometry. }\end{array}$ \\
\hline Ash et al., 2003[19] & $\begin{array}{l}\text { Country: Tanzania } \\
\text { Geography: rural } \\
\text { Population: M and F } 6-11 \text { years } \\
(n=830) \\
\text { Exclusion criteria: } \\
\text { ocular signs of xerophthalmia, } \\
\mathrm{Hb}<70 \mathrm{~g} / \mathrm{L} \text {, serious chronic disease }\end{array}$ & $\begin{array}{l}\text { Beverage reconstituted } \\
\text { from powder fortified } \\
\text { with: } 7 \text { vitamins and } \\
3 \text { minerals }\end{array}$ & $\begin{array}{l}\text { Iso-caloric non- } \\
\text { fortified beverage } \\
\text { reconstituted from } \\
\text { powder }\end{array}$ & 6 months & $\begin{array}{l}\mathrm{Hb} \text {, ferritin, erythrocyte } \\
\text { protoporphyrin, retinol, } \\
\text { anthropometry }\end{array}$ & $\begin{array}{l}\text { Change in all measured } \\
\text { biochemical and anthropometric } \\
\text { indices significantly greater in } \\
\text { fortified group. }\end{array}$ \\
\hline
\end{tabular}


Table 2. Cont

\begin{tabular}{|c|c|c|c|c|c|c|}
\hline Reference & Population & Intervention & Control & Duration & Outcomes & Main Results \\
\hline $\begin{array}{l}\text { Hyder } \text { et al., } \\
2007[15]\end{array}$ & $\begin{array}{l}\text { Country: Bangladesh } \\
\text { Geography: rural } \\
\text { Population: F } 12 \pm 1.9 \text { years }(n=1125) \\
\text { Exclusion criteria: } \\
\mathrm{Hb}<70 \mathrm{~g} / \mathrm{L} \text {, night blindness, goiter, } \\
\text { acute illness }\end{array}$ & $\begin{array}{l}\text { Beverage reconstituted } \\
\text { from powder fortified } \\
\text { with: } 8 \text { vitamins and } \\
3 \text { minerals }\end{array}$ & $\begin{array}{l}\text { Iso-caloric non-fortified } \\
\text { beverage powder }\end{array}$ & 6 months & $\begin{array}{l}\mathrm{Hb} \text {, ferritin, } \\
\text { retinol, zinc, } \\
\text { anthropometry }\end{array}$ & $\begin{array}{l}\text { Change in } \mathrm{Hb} \text {, ferritin, retinol, and } \\
\text { anthropometry significantly greater } \\
\text { in fortified group from } 0-6 \text { months, } \\
\text { but not from } 6-12 \text { months. No } \\
\text { significant difference between groups } \\
\text { in zinc. }\end{array}$ \\
\hline $\begin{array}{l}\text { Makola et al., } \\
\text { 2003[21] }\end{array}$ & $\begin{array}{l}\text { Country: Tanzania } \\
\text { Geography: rural } \\
\text { Population: pregnant women }(n=439) \\
\text { Exclusion criteria: } \\
\text { Gestation }<12 \text { or }>34 \text { weeks, } \\
\mathrm{Hb}<80 \mathrm{~g} / \mathrm{L} \text {, serious medical condition } \\
\text { or pregnancy complication }\end{array}$ & $\begin{array}{l}\text { Beverage reconstituted } \\
\text { from powder fortified } \\
\text { with: } 8 \text { vitamins and } \\
3 \text { minerals }\end{array}$ & $\begin{array}{l}\text { Iso-caloric non-fortified } \\
\text { beverage powder for home } \\
\text { reconstitution }\end{array}$ & 8 weeks & $\begin{array}{l}\mathrm{Hb} \text {, ferritin, } \\
\text { retinol, thyroid } \\
\text { stimulating } \\
\text { hormone }\end{array}$ & $\begin{array}{l}\text { Change in } \mathrm{Hb} \text { and ferritin } \\
\text { significantly greater in fortified } \\
\text { group. No significant difference } \\
\text { between groups in retinol and } \\
\text { thyroid stimulating hormone. }\end{array}$ \\
\hline $\begin{array}{l}\text { Solon et al., } \\
2003[20]\end{array}$ & $\begin{array}{l}\text { Country: Philippines } \\
\text { Geography: urban } \\
\text { Age: } \mathrm{M} \text { and } \mathrm{F} \text { grade } 1-6, \text { mean age } \\
9.9 \pm 2.2 \text { years }(n=831) \\
\text { Exclusion criteria: } \mathrm{Hb} \leq 80 \mathrm{~g} / \mathrm{L}\end{array}$ & $\begin{array}{l}\text { Beverage reconstituted } \\
\text { from powder fortified } \\
\text { with: } 8 \text { vitamins and } \\
3 \text { minerals }\end{array}$ & $\begin{array}{l}\text { Iso-caloric non-fortified } \\
\text { beverage reconstituted from } \\
\text { powder }\end{array}$ & 16 weeks & $\begin{array}{l}\mathrm{Hb} \text {, urinary } \\
\text { iodine, } \\
\text { anthropometry, } \\
\text { physical fitness, } \\
\text { cognitive } \\
\text { performance } \\
\end{array}$ & $\begin{array}{l}\text { Change in iodine significantly greater } \\
\text { in fortified group. No significant } \\
\text { difference between groups in } \mathrm{Hb} \text {, } \\
\text { anthropometry, physical fitness, or } \\
\text { cognitive performance. }\end{array}$ \\
\hline $\begin{array}{l}\text { Taljaard et al., } \\
\text { 2013[14] }\end{array}$ & $\begin{array}{l}\text { Country: South Africa } \\
\text { Geography: peri-urban } \\
\text { Age: M and F } 6-11 \text { years }(n=414) \\
\text { Exclusion criteria: health condition } \\
\text { precluding cognitive testing, medication } \\
\text { or supplement use }\end{array}$ & $\begin{array}{l}\text { Beverage reconstituted } \\
\text { from powder fortified } \\
\text { with: } 8 \text { vitamins and } \\
4 \text { minerals (with or } \\
\text { without nutritive } \\
\text { sweetener; group } \\
\text { receiving sweetened } \\
\text { beverage included in } \\
\text { meta-analysis) }\end{array}$ & $\begin{array}{l}\text { Iso-caloric non-fortified } \\
\text { beverage reconstituted from } \\
\text { powder (with or without } \\
\text { nutritive sweetener; group } \\
\text { receiving sweetened beverage } \\
\text { included in meta-analysis) }\end{array}$ & 8.5 months & $\begin{array}{l}\mathrm{Hb} \text {, ferritin, } \\
\text { transferrin } \\
\text { receptors, zinc } \\
\text { protoporphyrin, } \\
\text { retinol, zinc, } \\
\text { anthropometry, } \\
\text { cognitive } \\
\text { performance }\end{array}$ & $\begin{array}{l}\text { Change in } \mathrm{Hb} \text {, ferritin, zinc } \\
\text { protoporphyrin, and some cognitive } \\
\text { performance indicators significantly } \\
\text { greater in MMN fortified groups; } \\
\text { MMN groups w/ significantly } \\
\text { decreased odds of iron deficiency. No } \\
\text { significant difference between groups } \\
\text { in transferrin receptors, retinol or zinc. }\end{array}$ \\
\hline
\end{tabular}


Table 2. Cont

\begin{tabular}{|c|c|c|c|c|c|c|}
\hline Reference & Population & Intervention & Control & Duration & Outcomes & Main Results \\
\hline $\begin{array}{l}\text { Thankachan et al., } \\
\text { 2012[11] }\end{array}$ & $\begin{array}{l}\text { Country: India } \\
\text { Geography: urban } \\
\text { Population: M and } \mathrm{F} 6-12 \text { years } \\
(n=246) \\
\text { Exclusion criteria: } \mathrm{Hb}<80 \mathrm{~g} / \mathrm{L}, \text { chronic } \\
\text { illness, physical handicaps, WAZ or } \\
\mathrm{HAZ}<-3\end{array}$ & $\begin{array}{l}\text { Beverage reconstituted } \\
\text { from powder fortified } \\
\text { with: } 5 \text { vitamins and } \\
2 \text { minerals }\end{array}$ & $\begin{array}{l}\text { Iso-caloric non-fortified } \\
\text { beverage reconstituted from } \\
\text { powder }\end{array}$ & 8 weeks & $\begin{array}{l}\text { Hb, ferritin, } \\
\text { transferrin } \\
\text { receptors, zinc } \\
\text { protoporphyrin, } \\
\text { retinol, zinc, } \\
\text { vitamin B12, } \\
\text { RBC folate, } \\
\text { body iron } \\
\text { stores, vitamin } \\
\text { C, } \\
\text { anthropometry, } \\
\text { morbidity }\end{array}$ & $\begin{array}{l}\text { Change in } \mathrm{Hb} \text {, ferritin, transferrin } \\
\text { receptors, zinc protoporphyrin, } \\
\text { retinol, vitamin } \mathrm{B} 12 \text {, folate, body } \\
\text { iron stores and vitamin C } \\
\text { significantly greater in fortified } \\
\text { group. No significant difference } \\
\text { between groups in zinc, } \\
\text { anthropometry, or morbidity. }\end{array}$ \\
\hline $\begin{array}{l}\text { Vaz et al., } \\
2011[13]\end{array}$ & $\begin{array}{l}\text { Country: India } \\
\text { Geography: urban } \\
\text { Population: M and F 7-10.5 years } \\
(n=190) \\
\text { Exclusion criteria: } \\
\mathrm{Hb}<80 \mathrm{~g} / \mathrm{L} \text {, cardiovascular or } \\
\text { respiratory disease, } \\
\text { physical disability, } \\
\text { recent history of serious infections, } \\
\text { surgery, or injuries, nutritional } \\
\text { supplements use }\end{array}$ & $\begin{array}{l}\text { Beverage reconstituted } \\
\text { from powder fortified } \\
\text { with: } 11 \text { vitamins and } 6 \\
\text { minerals }\end{array}$ & $\begin{array}{l}\text { Iso-caloric non-fortified } \\
\text { beverage reconstituted from } \\
\text { powder, non-intervention } \\
\text { control (placebo control only } \\
\text { included in meta-analysis) }\end{array}$ & 4 months & $\begin{array}{l}\text { Ferritin, } \\
\text { transferrin } \\
\text { receptors, } \\
\text { vitamin } \mathrm{B} 12, \\
\text { vitamin } \mathrm{C}, \mathrm{RBC} \\
\text { thiamin, folate, } \\
\text { and riboflavin, } \\
\text { pyridoxal } \\
\text { phosphate, } \\
\text { niacin, aerobic } \\
\text { capacity, whole } \\
\text { body endurance }\end{array}$ & $\begin{array}{l}\text { Change in ferritin, transferrin } \\
\text { receptors, vitamin B12, vitamin C, } \\
\text { pyridoxal phosphate, RBC thiamin, } \\
\text { folate, and riboflavin, aerobic } \\
\text { capacity, and whole body endurance } \\
\text { significantly greater in fortified group. } \\
\text { No significant difference between } \\
\text { groups in niacin. }\end{array}$ \\
\hline
\end{tabular}


Table 3. Methodological quality of studies included in the analysis.

\begin{tabular}{|c|c|c|c|c|c|c|c|c|c|}
\hline $\begin{array}{l}\text { Author, } \\
\text { year }\end{array}$ & $\begin{array}{c}\text { Adequate } \\
\text { Sequence } \\
\text { Generation? }\end{array}$ & $\begin{array}{c}\text { Adequate } \\
\text { Allocation } \\
\text { Concealment? }\end{array}$ & Blinding & $\begin{array}{c}\text { Loss to } \\
\text { Follow-Up }\end{array}$ & $\begin{array}{c}\text { Intention } \\
\text { to Treat } \\
\text { Analysis? }\end{array}$ & $\begin{array}{c}\text { Free of } \\
\text { Selective } \\
\text { Reporting? }\end{array}$ & Other Bias? & Comments & Grade \\
\hline $\begin{array}{l}\text { Aaron, } \\
2011[17]\end{array}$ & $\begin{array}{l}\text { Yes, groups } \\
\text { stratified } \\
\text { proportionate to } \\
\text { number of students } \\
\text { in each school and } \\
\text { class level }\end{array}$ & $\begin{array}{l}\text { Yes, beverages } \\
\text { identical in } \\
\text { taste and } \\
\text { appearance }\end{array}$ & $\begin{array}{l}\text { Double } \\
\text { blind, } \\
\text { placebo } \\
\text { controlled }\end{array}$ & $\begin{array}{l}6 \%(n=32) \text { due } \\
\text { to relocation } \\
\text { or school } \\
\text { withdrawal }\end{array}$ & Yes & Yes & 2 schools & $\begin{array}{l}\text { Beverage contained } \\
\text { maize, soy isolate and } \\
\text { bioflavonoids in addition to } \\
\text { MMN, de-worming } 1 \\
\text { month prior to end point } \\
\text { per school policy }\end{array}$ & High \\
\hline $\begin{array}{l}\text { Abrams, } \\
2003[18]\end{array}$ & $\begin{array}{l}\text { No, students in } \\
2 \text { schools assigned } \\
\text { to intervention and } \\
\text { control groups, } \\
\text { respectively }\end{array}$ & $\begin{array}{l}\text { Yes, beverages } \\
\text { identical in } \\
\text { flavor and } \\
\text { appearance }\end{array}$ & $\begin{array}{l}\text { Double } \\
\text { blind, } \\
\text { placebo } \\
\text { controlled }\end{array}$ & $\begin{array}{l}15 \%(n=44) \\
\text { reasons for loss } \\
\text { not stated }\end{array}$ & Yes & Yes & $\begin{array}{l}\text { Lack of } \\
\text { participant-level } \\
\text { randomization }\end{array}$ & $\begin{array}{l}\beta \text {-carotene rather than } \\
\text { retinol as source of vitamin } \\
\text { A, short ( } 8 \text { week) } \\
\text { intervention period }\end{array}$ & $\begin{array}{l}\text { Moderate } \\
\text { (inadequate } \\
\text { sequence } \\
\text { generation) }\end{array}$ \\
\hline $\begin{array}{l}\text { Angeles- } \\
\text { Agdeppa, } \\
2011[10]\end{array}$ & $\begin{array}{l}\text { Detailed method } \\
\text { not stated. }\end{array}$ & $\begin{array}{l}\text { Not stated } \\
\text { directly, } \\
\text { beverages } \\
\text { provided in foil } \\
\text { packs }\end{array}$ & $\begin{array}{l}\text { Double blind } \\
\text { placebo } \\
\text { controlled }\end{array}$ & $\begin{array}{l}11 \%(n=11) \\
\text { due to } \\
\text { relocation, } \\
\text { school change, } \\
\text { or absence } \\
\text { during data } \\
\text { collection }\end{array}$ & Not stated & Yes & & $\begin{array}{l}\text { Non-fortified juice } \\
\text { contained vitamin } \mathrm{C} \text {. All } \\
\text { children anemic at baseline } \\
(\mathrm{Hb}>70 \text { and }<120 \mathrm{~g} / \mathrm{L}) \text {, } \\
\text { all dewormed } 1 \text { week prior } \\
\text { to intervention }\end{array}$ & $\begin{array}{l}\text { Low (method of } \\
\text { randomization } \\
\text { not reported, all } \\
\text { children } \\
\text { moderately } \\
\text { anemic at } \\
\text { baseline) }\end{array}$ \\
\hline $\begin{array}{l}\text { Ash, } \\
\text { 2003[19] }\end{array}$ & $\begin{array}{l}\text { Method not stated, } \\
\text { students stratified } \\
\text { by median } \mathrm{Hb} \\
\text { prior to } \\
\text { randomization }\end{array}$ & $\begin{array}{l}\text { Yes, beverages } \\
\text { identical in } \\
\text { taste and } \\
\text { appearance }\end{array}$ & $\begin{array}{l}\text { Double } \\
\text { blind, } \\
\text { placebo } \\
\text { controlled }\end{array}$ & $\begin{array}{l}7 \%(n=56) \text { due } \\
\text { to poor } \\
\text { attendance, } \\
\text { leaving school, } \\
\text { refusing } \\
\text { venipuncture }\end{array}$ & Yes & Yes & $\begin{array}{l}\text { Baseline ferritin } \\
\text { differed } \\
\text { significantly } \\
\text { between groups }\end{array}$ & -- & $\begin{array}{l}\text { Moderate } \\
\text { (method of } \\
\text { randomization } \\
\text { not reported) }\end{array}$ \\
\hline
\end{tabular}


Table 3. Cont

\begin{tabular}{|c|c|c|c|c|c|c|c|c|c|}
\hline $\begin{array}{l}\text { Author, } \\
\text { year }\end{array}$ & $\begin{array}{c}\text { Adequate } \\
\text { Sequence } \\
\text { Generation? }\end{array}$ & $\begin{array}{c}\text { Adequate } \\
\text { Allocation } \\
\text { Concealment? }\end{array}$ & Blinding & $\begin{array}{c}\text { Loss to } \\
\text { Follow-Up }\end{array}$ & $\begin{array}{c}\text { Intention } \\
\text { to Treat } \\
\text { Analysis? }\end{array}$ & $\begin{array}{c}\text { Free of } \\
\text { Selective } \\
\text { Reporting? }\end{array}$ & Other Bias? & Comments & Grade \\
\hline $\begin{array}{l}\text { Hyder, } \\
2007[15]\end{array}$ & $\begin{array}{l}\text { Yes , random \# } \\
\text { assigned to } \\
\text { participants, } \\
\text { groups defined as } \\
\text { even and odd }\end{array}$ & $\begin{array}{l}\text { Yes, beverages } \\
\text { identical in } \\
\text { weight, color, } \\
\text { flavor and } \\
\text { appearance }\end{array}$ & $\begin{array}{l}\text { Double } \\
\text { blind, } \\
\text { placebo } \\
\text { controlled }\end{array}$ & $\begin{array}{l}12 \%(n=136) \\
\text { due to missing } \\
\text { data, illness, } \\
\text { refusal }\end{array}$ & Yes & Yes & $\begin{array}{l}\text { Parasitic infection } \\
\text { not measured, } \\
\text { analysis involved } \\
\text { females only }\end{array}$ & $\begin{array}{l}\text { Energy content of } \\
\text { intervention/placebo not } \\
\text { reported, target population } \\
\text { adolescent girls, 0-6 month } \\
\text { intervention period } \\
\text { included in meta-analysis }\end{array}$ & $\begin{array}{l}\text { Moderate } \\
\text { (included F only } \\
\text { so not } \\
\text { generalizable) }\end{array}$ \\
\hline $\begin{array}{l}\text { Makola, } \\
\text { 2003[21] }\end{array}$ & $\begin{array}{l}\text { Yes, block } \\
\text { randomization } \\
\text { (10 subjects per } \\
\text { block) at each of } \\
6 \text { study centers }\end{array}$ & $\begin{array}{l}\text { Yes, beverages } \\
\text { identical in } \\
\text { appearance, } \\
\text { color and taste } \\
\text { and packaged } \\
\text { similarly }\end{array}$ & $\begin{array}{l}\text { Double } \\
\text { blind, } \\
\text { placebo } \\
\text { controlled }\end{array}$ & $\begin{array}{l}41 \%(n=180) \\
\text { due to logistic } \\
\text { problems, early } \\
\text { delivery }\end{array}$ & Not stated & Yes & & $\begin{array}{l}\text { 2nd-3rd trimester pregnant } \\
\text { women included, short } \\
\text { ( } 8 \text { week) intervention } \\
\text { period. Variable } \\
\text { simultaneous use of } \\
\text { iron/folic acid supplements }\end{array}$ & $\begin{array}{l}\text { Moderate (high } \\
\text { loss to follow-up) }\end{array}$ \\
\hline $\begin{array}{l}\text { Solon, } \\
2003[20]\end{array}$ & $\begin{array}{l}\text { Not stated, students } \\
\text { randomized into } \\
4 \text { groups } \\
(2 * 2 \text {, beverage* } \\
\text { de-worming tablets) }\end{array}$ & $\begin{array}{l}\text { Yes, beverages } \\
\text { indistinguishab } \\
\text { le in } \\
\text { appearance, } \\
\text { smell, and taste }\end{array}$ & $\begin{array}{l}\text { Double } \\
\text { blind, } \\
\text { placebo } \\
\text { controlled }\end{array}$ & $\begin{array}{l}5 \%(n=43), \\
\text { reasons not } \\
\text { described }\end{array}$ & Not stated & Yes & $\begin{array}{l}\text { Approximately half } \\
\text { of subjects in } \\
\text { fortified and } \\
\text { placebo beverage } \\
\text { group received de- } \\
\text { worming treatment. }\end{array}$ & $\begin{array}{l}\text { Effects on micronutrient } \\
\text { status other than iron and } \\
\text { iodine not reported. All } \\
\text { participants included in } \\
\text { meta-analysis by } \\
\text { beverage group. }\end{array}$ & $\begin{array}{l}\text { Moderate } \\
\text { (method of } \\
\text { randomization } \\
\text { not reported) }\end{array}$ \\
\hline $\begin{array}{l}\text { Taljaard, } \\
\text { 2013[14] }\end{array}$ & $\begin{array}{l}\text { Not stated, } \\
\text { randomisation to } \\
4 \text { groups ( } 2 * 2 \\
\text { MMN*sugar) by } \\
\text { school, classroom, } \\
\text { and gender }\end{array}$ & $\begin{array}{l}\text { Yes, beverages } \\
\text { identical in } \\
\text { color and taste. }\end{array}$ & $\begin{array}{l}\text { Double } \\
\text { blind, } \\
\text { placebo } \\
\text { controlled }\end{array}$ & $\begin{array}{l}3.9 \%(n=16) \\
\text { due to leaving } \\
\text { school }\end{array}$ & Not stated & Yes & & $\begin{array}{l}\text { Only iso-caloric MMN + } \\
\text { sugar and sugar only } \\
\text { interventions included in } \\
\text { meta-analysis. De-worming } \\
\text { prior to intervention }\end{array}$ & High \\
\hline
\end{tabular}


Table 3. Cont

\begin{tabular}{|c|c|c|c|c|c|c|c|c|c|}
\hline $\begin{array}{l}\text { Author, } \\
\text { year }\end{array}$ & $\begin{array}{c}\text { Adequate } \\
\text { Sequence } \\
\text { Generation? }\end{array}$ & $\begin{array}{c}\text { Adequate } \\
\text { Allocation } \\
\text { Concealment? }\end{array}$ & Blinding & $\begin{array}{c}\text { Loss to } \\
\text { Follow-Up }\end{array}$ & $\begin{array}{c}\text { Intention } \\
\text { to Treat } \\
\text { Analysis? }\end{array}$ & $\begin{array}{c}\text { Free of } \\
\text { Selective } \\
\text { Reporting? }\end{array}$ & Other Bias? & Comments & Grade \\
\hline $\begin{array}{l}\text { Thankachan, } \\
\text { 2012[11] }\end{array}$ & $\begin{array}{l}\text { Yes, block } \\
\text { randomization } \\
\text { with a computer- } \\
\text { generated list in } \\
\text { blocks of } 20\end{array}$ & $\begin{array}{l}\text { Yes, beverages } \\
\text { identical in } \\
\text { color, size, } \\
\text { and taste }\end{array}$ & $\begin{array}{l}\text { Double } \\
\text { blind, } \\
\text { placebo } \\
\text { controlled }\end{array}$ & $\begin{array}{l}1 \%(n=3) \text { due } \\
\text { to prolonged } \\
\text { school absence } \\
\text { or refusal of } \\
\text { blood draw }\end{array}$ & Not stated & Yes & $\begin{array}{l}\text { Included only } \\
\text { participants who } \\
\text { were iron deficient } \\
\text { at baseline (serum } \\
\text { ferritin }<45 \mathrm{pmol} / \mathrm{L} \text { ) }\end{array}$ & $\begin{array}{l}\text { Short ( } 8 \text { week) } \\
\text { intervention period }\end{array}$ & $\begin{array}{l}\text { Moderate } \\
\text { (included only } \\
\text { iron-deficient } \\
\text { participants, not } \\
\text { generalizable) }\end{array}$ \\
\hline $\begin{array}{l}\text { Vaz, } \\
2011[13]\end{array}$ & $\begin{array}{l}\text { Yes, block } \\
\text { randomization } \\
\text { using computer } \\
\text { generated } \\
\text { sequence into } \\
3 \text { arms (MMN } \\
\text { fortified, } \\
\text { unfortified, and no } \\
\text { beverage) }\end{array}$ & $\begin{array}{l}\text { Not stated, } \\
\text { MMN fortified } \\
\text { and unfortified } \\
\text { beverages both } \\
\text { choco-malt }\end{array}$ & $\begin{array}{l}\text { Double blind } \\
\text { placebo } \\
\text { controlled }\end{array}$ & $\begin{array}{l}4 \%(n=13) \text { due } \\
\text { to withdrawal } \\
\text { of consent }\end{array}$ & Yes & Yes & $\begin{array}{l}\text { Baseline ferritin } \\
\text { differed } \\
\text { significantly } \\
\text { between MMN } \\
\text { fortified and } \\
\text { unfortified } \\
\text { beverage groups }\end{array}$ & $\begin{array}{l}\text { Only iso-caloric MMN } \\
\text { fortified and unfortified } \\
\text { interventions included in } \\
\text { meta-analysis }\end{array}$ & $\begin{array}{l}\text { Moderate } \\
\text { (allocation } \\
\text { concealment not } \\
\text { reported directly) }\end{array}$ \\
\hline
\end{tabular}


Table 4. Summary of findings and overall assessment of quality of evidence grade by study outcome.

\begin{tabular}{|c|c|c|c|c|c|c|c|c|}
\hline \multicolumn{6}{|c|}{ Quality Assessment } & \multicolumn{3}{|c|}{ Summary of Findings } \\
\hline $\begin{array}{c}\text { No. } \\
\text { Studies }\end{array}$ & $\begin{array}{c}I^{2} \\
(\%)\end{array}$ & Heterogeneity & $\begin{array}{c}\text { Generalizable to } \\
\text { Population of Interest? }\end{array}$ & $\begin{array}{c}\text { Generalizable to } \\
\text { Intervention of Interest? }\end{array}$ & $\begin{array}{c}\text { Other Sources of Bias (e.g., } \\
\text { Major Limitations in Study } \\
\text { Design) }\end{array}$ & $\begin{array}{c}\text { No. } \\
\text { Participants }\end{array}$ & $\begin{array}{c}\text { Publication } \\
\text { Bias r } \\
(p \text {-value })\end{array}$ & $\begin{array}{c}\text { Effect } \\
\text { Estimate }\end{array}$ \\
\hline \multicolumn{9}{|c|}{ Hemoglobin $(\mathrm{g} / \mathrm{L})$ : Overall quality of evidence grade $=$ moderate } \\
\hline 8 & 92 & $\begin{array}{l}6 \text { of } 8 \text { studies found } \\
\text { significantly greater } \\
\text { increase in } \mathrm{Hb} \text { in } \\
\text { intervention group. Other } \\
\text { studies found no difference } \\
\text { between groups. }\end{array}$ & $\begin{array}{l}7 \text { of } 8 \text { studies conducted in } \\
\mathrm{M} \text { and } \mathrm{F} \text { school age children } \\
\text { in lower-income countries. } 1 \\
\text { study in F adolescents in } \\
\text { Bangladesh. }\end{array}$ & $\begin{array}{l}\text { Variability in micronutrient } \\
\text { composition, dose, and } \\
\text { duration. Reconstituted } \\
\text { powder used in } 6 \text { of } 8 \\
\text { studies. }\end{array}$ & $\begin{array}{l}\text { Study w/highest iron dose found } \\
\text { no difference between groups, } \\
\text { several randomized by school, } \\
\text { de-worming protocols } \\
\text { inconsistent. }\end{array}$ & 3835 & $0.25(0.56)$ & $\begin{array}{c}2.76 \\
{[1.19,4.33]}\end{array}$ \\
\hline \multicolumn{9}{|c|}{ Ferritin $(\mathrm{pmol} / \mathrm{L})$ : Overall quality of evidence grade $=$ moderate } \\
\hline 8 & 95 & $\begin{array}{l}6 \text { of } 8 \text { studies found } \\
\text { significantly greater } \\
\text { increase in ferritin in } \\
\text { intervention group. Other } \\
\text { studies found no significant } \\
\text { difference between groups. }\end{array}$ & $\begin{array}{l}7 \text { of } 8 \text { studies conducted in } \\
\mathrm{M} \text { and } \mathrm{F} \text { school age children } \\
\text { in lower-income countries. } 1 \\
\text { study in F adolescents in } \\
\text { Bangladesh. }\end{array}$ & $\begin{array}{l}\text { Variability in micronutrient } \\
\text { composition, dose, and } \\
\text { duration. Reconstituted } \\
\text { powder used in } 6 \text { of } 8 \\
\text { studies. }\end{array}$ & $\begin{array}{l}\text { Study w/highest iron dose found } \\
\text { no difference between groups, } \\
\text { baseline ferritin differed in } 1 \\
\text { study, several randomized by } \\
\text { school, de-worming protocols } \\
\text { inconsistent. }\end{array}$ & 3891 & $0.94(0.004)$ & $\begin{array}{c}15.42 \\
{[5.73,25.12]}\end{array}$ \\
\hline \multicolumn{9}{|c|}{ Retinol $(\mu \mathrm{mol} / \mathrm{L})$ : Overall quality of evidence grade $=$ low } \\
\hline 5 & 61 & $\begin{array}{l}3 \text { of } 5 \text { studies found } \\
\text { significantly greater } \\
\text { increase in retinol in } \\
\text { intervention group. Other } \\
\text { studies found no significant } \\
\text { difference between groups. }\end{array}$ & $\begin{array}{l}4 \text { of } 5 \text { studies conducted in } \\
\mathrm{M} \text { and } \mathrm{F} \text { school age children } \\
\text { in lower-income countries. } 1 \\
\text { study in F adolescents in } \\
\text { Bangladesh. }\end{array}$ & $\begin{array}{l}\text { Variability in micronutrient } \\
\text { composition, dose, and } \\
\text { duration. Reconstituted } \\
\text { powder used in } 4 \text { of } 5 \\
\text { studies. }\end{array}$ & $\begin{array}{l}\text { Randomization method unclear or } \\
\text { at school level in some studies, de- } \\
\text { worming protocols inconsistent. }\end{array}$ & 2049 & $0.30(0.62)$ & $\begin{array}{c}0.05 \\
{[-0.03,0.13]}\end{array}$ \\
\hline
\end{tabular}


Table 4. Cont

\begin{tabular}{|c|c|c|c|c|c|c|c|c|}
\hline \multicolumn{6}{|c|}{ Quality Assessment } & \multicolumn{3}{|c|}{ Summary of Findings } \\
\hline $\begin{array}{l}\text { No. } \\
\text { Studies }\end{array}$ & $\begin{array}{c}I^{2} \\
(\%)\end{array}$ & Heterogeneity & $\begin{array}{c}\text { Generalizable to } \\
\text { Population of Interest? }\end{array}$ & $\begin{array}{c}\text { Generalizable to } \\
\text { Intervention of Interest? }\end{array}$ & $\begin{array}{c}\text { Other Sources of Bias (e.g., } \\
\text { Major Limitations in Study } \\
\text { Design) }\end{array}$ & $\begin{array}{c}\text { No. } \\
\text { Participants }\end{array}$ & $\begin{array}{l}\text { Publication } \\
\text { Bias r } \\
(p \text {-value })\end{array}$ & $\begin{array}{c}\text { Effect } \\
\text { Estimate }\end{array}$ \\
\hline \multicolumn{9}{|c|}{ Zinc $(\mu \mathrm{mol} / \mathrm{L})$ : Overall quality of evidence grade $=$ low } \\
\hline 4 & 75 & $\begin{array}{l}2 \text { of } 4 \text { studies found } \\
\text { significantly greater } \\
\text { increase in zinc in } \\
\text { intervention group. Other } \\
\text { studies found no significant } \\
\text { difference between groups. }\end{array}$ & $\begin{array}{l}3 \text { of } 4 \text { studies conducted in } \\
M \text { and F school age children } \\
\text { in lower-income countries. } 1 \\
\text { study in F adolescents in } \\
\text { Bangladesh. }\end{array}$ & $\begin{array}{l}\text { Variability in micronutrient } \\
\text { composition, dose, and } \\
\text { duration. Reconstituted } \\
\text { powder used in } 2 \text { of } 4 \\
\text { studies. }\end{array}$ & $\begin{array}{l}\text { Randomization method not } \\
\text { explicit in } 2 \text { studies, de-worming } \\
\text { protocols inconsistent. }\end{array}$ & 1690 & $0.09(0.91)$ & $\begin{array}{c}0.92 \\
{[-1.45,3.30]}\end{array}$ \\
\hline \multicolumn{9}{|c|}{ Vitami n B12 (pmol/L): Overall quality of evidence grade = very low } \\
\hline 3 & 99 & $\begin{array}{l}2 \text { of } 3 \text { studies found } \\
\text { significantly greater } \\
\text { increase in vitamin B12 in } \\
\text { intervention group. Other } \\
\text { study found no differences } \\
\text { between groups. }\end{array}$ & $\begin{array}{l}\text { All studies conducted in } \mathrm{M} \\
\text { and } \mathrm{F} \text { school children in } \\
\text { lower-income countries. }\end{array}$ & $\begin{array}{l}\text { Variability in micronutrient } \\
\text { composition, dose, and } \\
\text { duration. Reconstituted } \\
\text { powder used in all studies }\end{array}$ & $\begin{array}{l}\text { One study randomized by school, } \\
\text { infection and/or parasites not } \\
\text { treated or measured consistently. }\end{array}$ & 644 & $0.91(0.27)$ & $\begin{array}{c}96.2 \\
{[-142.2,334.6]}\end{array}$ \\
\hline \multicolumn{9}{|c|}{ Weight $(\mathrm{kg})$ : Overall quality of evidence grade $=$ moderate } \\
\hline 6 & 85 & $\begin{array}{l}3 \text { of } 6 \text { studies found } \\
\text { significantly greater } \\
\text { increase in vitamin B12 in } \\
\text { intervention group. Other } \\
\text { studies found no } \\
\text { differences between } \\
\text { groups. }\end{array}$ & $\begin{array}{l}5 \text { of } 6 \text { studies conducted in } \\
M \text { and F school age children } \\
\text { in lower-income countries. } 1 \\
\text { study in F adolescents in } \\
\text { Bangladesh. }\end{array}$ & $\begin{array}{l}\text { Variability in micronutrient } \\
\text { composition, dose, and } \\
\text { duration. Reconstituted } \\
\text { powder used in all studies. }\end{array}$ & $\begin{array}{l}\text { Randomization method unclear or } \\
\text { at school level in } 4 \text { studies, de- } \\
\text { worming protocols inconsistent. }\end{array}$ & 2977 & $0.04(0.94)$ & $0.30[0.01,0.58]$ \\
\hline
\end{tabular}


Table 4. Cont.

\begin{tabular}{|c|c|c|c|c|c|c|c|c|}
\hline \multicolumn{6}{|c|}{ Quality Assessment } & \multicolumn{3}{|c|}{ Summary of Findings } \\
\hline $\begin{array}{c}\text { No. } \\
\text { Studies }\end{array}$ & $\begin{array}{c}I^{2} \\
(\%)\end{array}$ & Heterogeneity & $\begin{array}{c}\text { Generalizable to } \\
\text { Population of Interest? }\end{array}$ & $\begin{array}{c}\text { Generalizable to } \\
\text { Intervention of Interest? }\end{array}$ & $\begin{array}{c}\text { Other Sources of Bias (e.g., } \\
\text { Major Limitations in Study } \\
\text { Design) }\end{array}$ & $\begin{array}{c}\text { No. } \\
\text { Participants }\end{array}$ & $\begin{array}{c}\text { Publication } \\
\text { Bias r } \\
\text { (p-value) }\end{array}$ & $\begin{array}{c}\text { Effect } \\
\text { Estimate }\end{array}$ \\
\hline \multicolumn{9}{|c|}{ Height $(\mathrm{cm})$ : Overall quality of evidence grade $=$ moderate } \\
\hline 5 & 78 & $\begin{array}{l}2 \text { of } 5 \text { studies found } \\
\text { significantly greater } \\
\text { increase in vitamin B12 in } \\
\text { intervention group. Other } \\
\text { studies found no } \\
\text { differences between } \\
\text { groups. }\end{array}$ & $\begin{array}{l}4 \text { of } 5 \text { studies conducted in } \\
\mathrm{M} \text { and } \mathrm{F} \text { school age children } \\
\text { in lower-income countries. } 1 \\
\text { study in F adolescents in } \\
\text { Bangladesh. }\end{array}$ & $\begin{array}{l}\text { Variability in micronutrient } \\
\text { composition, dose, and } \\
\text { duration. Reconstituted } \\
\text { powder used in } 4 \text { of } 5 \\
\text { studies. }\end{array}$ & $\begin{array}{l}\text { Randomization not outlined } \\
\text { specifically in } 3 \text { studies, } \\
\text { de-worming protocols } \\
\text { inconsistent. }\end{array}$ & 2697 & $0.12(0.85)$ & $\begin{array}{c}0.17 \\
{[-0.16,0.50]}\end{array}$ \\
\hline \multicolumn{9}{|c|}{ Weight-for-age (Z): Overall quality of evidence grade $=$ low } \\
\hline 4 & 59 & $\begin{array}{l}2 \text { of } 4 \text { studies found } \\
\text { significantly greater } \\
\text { increase in vitamin B12 in } \\
\text { intervention group. Other } \\
\text { studies found no } \\
\text { differences between } \\
\text { groups. }\end{array}$ & $\begin{array}{l}\text { All studies conducted in } \mathrm{M} \\
\text { and F school children in } \\
\text { lower-income countries. }\end{array}$ & $\begin{array}{l}\text { Variability in micronutrient } \\
\text { composition, dose, and } \\
\text { duration. Reconstituted } \\
\text { powder used in } 3 \text { of } 4 \\
\text { studies. }\end{array}$ & $\begin{array}{l}\text { Method of randomization not } \\
\text { specific or at school level in } \\
\text { all studies, de-worming protocols } \\
\text { inconsistent. }\end{array}$ & 1385 & $0.65(0.35)$ & $\begin{array}{c}0.028 \\
{[-0.06,0.12]}\end{array}$ \\
\hline \multicolumn{9}{|c|}{ Height-for-age (Z): Overall quality of evidence grade $=$ moderate } \\
\hline 3 & 0 & $\begin{array}{l}\text { No significant differences } \\
\text { found between groups }\end{array}$ & $\begin{array}{l}\text { All studies conducted in } \mathrm{M} \\
\text { and F school children in } \\
\text { lower-income countries. }\end{array}$ & $\begin{array}{l}\text { Variability in micronutrient } \\
\text { composition, dose, and } \\
\text { duration. Reconstituted } \\
\text { powder used in } 2 \text { of } 3 \\
\text { studies. }\end{array}$ & $\begin{array}{l}\text { Method of randomization not } \\
\text { specific or at school level in all } \\
\text { studies, de-worming protocols } \\
\text { inconsistent. }\end{array}$ & 1124 & $0.10(0.94)$ & $\begin{array}{c}0.0 \\
{[-0.05,0.05]}\end{array}$ \\
\hline
\end{tabular}


Table 4. Cont

\begin{tabular}{|c|c|c|c|c|c|c|c|c|}
\hline \multicolumn{6}{|c|}{ Quality Assessment } & \multicolumn{3}{|c|}{ Summary of Findings } \\
\hline $\begin{array}{c}\text { No. } \\
\text { Studies }\end{array}$ & $\begin{array}{c}I^{2} \\
(\%)\end{array}$ & Heterogeneity & $\begin{array}{c}\text { Generalizable to } \\
\text { Population of Interest? }\end{array}$ & $\begin{array}{c}\text { Generalizable to } \\
\text { Intervention of Interest? }\end{array}$ & $\begin{array}{c}\text { Other Sources of Bias (e.g., } \\
\text { Major Limitations in Study } \\
\text { Design) }\end{array}$ & $\begin{array}{c}\text { No. } \\
\text { Participants }\end{array}$ & $\begin{array}{l}\text { Publication } \\
\text { Bias r } \\
(p \text {-value })\end{array}$ & $\begin{array}{c}\text { Effect } \\
\text { Estimate }\end{array}$ \\
\hline \multicolumn{9}{|c|}{ Anemia $(\mathrm{Hb}<110-120 \mathrm{~g} / \mathrm{L})$ : Overall quality of evidence grade $=$ moderate } \\
\hline 6 & 84 & $\begin{array}{l}5 \text { of } 6 \text { studies found } \\
\text { significantly greater } \\
\text { reduction in endpoint } \\
\text { prevalence of anemia in } \\
\text { intervention vs. control } \\
\text { group. }\end{array}$ & $\begin{array}{l}5 \text { of } 6 \text { studies conducted in } \\
\mathrm{M} \text { and } \mathrm{F} \text { children in lower- } \\
\text { income countries. } 1 \text { study in } \\
\mathrm{F} \text { adolescents in } \\
\text { Bangladesh. }\end{array}$ & $\begin{array}{l}\text { Variability in micronutrient } \\
\text { composition, dose, and } \\
\text { duration. Reconstituted } \\
\text { powder used in } 4 \text { of } 6 \\
\text { studies. }\end{array}$ & $\begin{array}{l}\text { Method of randomization not } \\
\text { specific or at school level in } \\
3 \text { studies, de-worming protocols } \\
\text { inconsistent. Study w/highest iron } \\
\text { dose only to find no } \\
\text { significant difference between } \\
\text { groups in } \\
\text { anemia reduction. }\end{array}$ & 2828 & $0.24(0.64)$ & $\begin{array}{c}\text { RR } 0.63 \\
{[0.54,0.73]}\end{array}$ \\
\hline \multicolumn{9}{|c|}{ Iron deficiency (ferritin $<27-45 \mathrm{pmol} / \mathrm{L}$ ): Overall quality of evidence grade $=$ moderate } \\
\hline 7 & 96 & $\begin{array}{l}4 \text { of } 7 \text { studies found } \\
\text { significantly greater } \\
\text { reduction in endpoint } \\
\text { prevalence of iron } \\
\text { deficiency in intervention } \\
v s . \text { control group }\end{array}$ & $\begin{array}{l}6 \text { of } 7 \text { studies conducted in } \\
\mathrm{M} \text { and } \mathrm{F} \text { children in lower- } \\
\text { income countries. } 1 \text { study in } \\
\mathrm{F} \text { adolescents in } \\
\text { Bangladesh. }\end{array}$ & $\begin{array}{l}\text { Variability in micronutrient } \\
\text { composition, dose, and } \\
\text { duration. Reconstituted } \\
\text { powder used in } 5 \text { of } 7 \\
\text { studies. }\end{array}$ & $\begin{array}{l}\text { Method of randomization not } \\
\text { specific or at school level in } \\
3 \text { studies, de-worming } \\
\text { protocols inconsistent. }\end{array}$ & 2523 & $0.23(0.62)$ & $\begin{array}{c}\text { RR } 0.32 \\
{[0.23,0.45]}\end{array}$ \\
\hline \multicolumn{9}{|c|}{ Iron deficiency anemia $(\mathrm{Hb}<110-120 \mathrm{~g} / \mathrm{L}$ and ferritin $<27-45 \mathrm{pmol} / \mathrm{L})$ : Overall quality of evidence grade $=$ low } \\
\hline 3 & 97 & $\begin{array}{l}2 \text { of } 3 \text { studies found } \\
\text { significantly greater } \\
\text { reduction in endpoint } \\
\text { prevalence of IDA in } \\
\text { intervention } v \text { s. control } \\
\text { group. }\end{array}$ & $\begin{array}{l}2 \text { of } 3 \text { studies conducted in } \\
\mathrm{M} \text { and } \mathrm{F} \text { children in lower- } \\
\text { income countries. } 1 \text { study in } \\
\mathrm{F} \text { adolescents } \\
\text { in Bangladesh. }\end{array}$ & $\begin{array}{l}\text { Variability in micronutrient } \\
\text { composition, dose, and } \\
\text { duration. Reconstituted } \\
\text { powder used in } 2 \text { of } 3 \\
\text { studies. }\end{array}$ & $\begin{array}{l}\text { De-worming protocols } \\
\text { inconsistent. }\end{array}$ & 1649 & $0.88(0.31)$ & $\begin{array}{c}\text { RR 0.13 } \\
{[0.07,0.25]}\end{array}$ \\
\hline
\end{tabular}




\subsection{Meta-Analysis}

Results of all trials conducted in children $(n=9$, total sample size $=4094)$ were included in the meta-analyses. Compared with controls who received a non-fortified beverage, children who received MMN-fortified beverages for a duration of 8 weeks to 6 months demonstrated significantly greater improvements in circulating hemoglobin $(+2.76 \mathrm{~g} / \mathrm{L}, 95 \%$-CI $[1.19,4.33], p=0.004 ; 8$ studies $)$ and ferritin $(+15.42 \mathrm{pmol} / \mathrm{L},[5.73,25.12], p=0.007 ; 8$ studies $)$. Exclusion of an anomalous study that found no change in hemoglobin or ferritin in either intervention or control groups did not significantly alter these results [17]. No significant intervention effects of fortified beverages were found for serum retinol $(+0.05 \mu \mathrm{mol} / \mathrm{L}[-0.03,0.13], p=0.16 ; 5$ studies $)$, zinc $(+0.92 \mu \mathrm{mol} / \mathrm{L}[-1.45,3.30], p=0.30$; 4 studies), or vitamin B12 (+96.2 pmol/L [-142.2, 334.6], $p=0.22 ; 3$ studies).

A moderate but significant effect was found for weight gain in the intervention compared with the control groups $(+0.30 \mathrm{~kg}$ [0.01,0.58], $p=0.04 ; 6$ studies), while neither height gain nor weight-for-age or height-for-age $Z$-scores differed significantly between groups $(+0.17 \mathrm{~cm}[-0.16,0.50], p=0.23$, 5 studies; +0.028 [ $-0.06,0.12], p=0.40,4$ studies; and $0.0[-0.05,0.05], p=0.98,3$ studies respectively). Although the number of data points precluded meta-analysis, we found some evidence of a favorable effect of supplementation with MMN-fortified beverages on riboflavin status [13,18], vitamin B6 [13], vitamin C [11,13], thiamin [13], folate [11,13], urinary iodine [7], body mass index [4,15], and mid-upper arm circumference $[4,15]$. For indices of physical performance results were inconsistent; one trial found significant improvement in aerobic capacity and endurance in intervention compared with control groups [13], while no difference in fitness index score or push up capacity between groups was found in a separate trial [7]. Two trials found no effects on cognitive indices [7,17], while one found gains in some cognitive test scores in the MMN-fortified group [14]. No effects were found on circulating niacin [13] or indices of morbidity [11].

Consumption of fortified beverages significantly reduced the risk of anemia (RR 0.63 [0.54, 0.73], $p<0.001 ; n=6$ studies, defined as $\mathrm{Hb}<110-120 \mathrm{~g} / \mathrm{L}$ ), iron deficiency (RR 0.32 [0.23,0.45], $p<0.001 ; n=7$ studies, defined as ferritin $<27-45 \mathrm{pmol} / \mathrm{L}$ ), and iron deficiency anemia (RR 0.13 [0.07, 0.25], $p<0.001 ; n=3$ studies, defined as a combination of biochemical criteria for iron deficiency and anemia) (Figure 1). Fortified beverage consumption did not significantly alter the risk of vitamin A or zinc deficiency.

Meta-regression analyses were conducted for outcomes with at least five data points from different trials (ferritin, hemoglobin and weight). Considered independently, daily iron dose, total iron dose (daily dose $\mathrm{x}$ duration), intervention duration, baseline status, and deworming (binary $\mathrm{Y} / \mathrm{N}$ ) were not significantly associated with the difference in ferritin change between groups. A significant inverse association was found between daily iron dose and hemoglobin change $(\beta=-0.4, p=0.02)$ with all studies included in analysis. The association was attenuated when total iron dose was substituted for daily iron dose $(\beta=-0.001, p=0.06)$. However, the significance of this relationship did not persist after excluding the study by Aaron et al. $(p>0.90)$ [17]. Baseline hemoglobin, intervention duration, and deworming were not predictive of change in hemoglobin, nor were baseline weight, intervention duration, or deworming predictive of differences in weight change. No adjustments were made for compliance rate, which was reported in only four studies. 
$1 \mathrm{~A}$

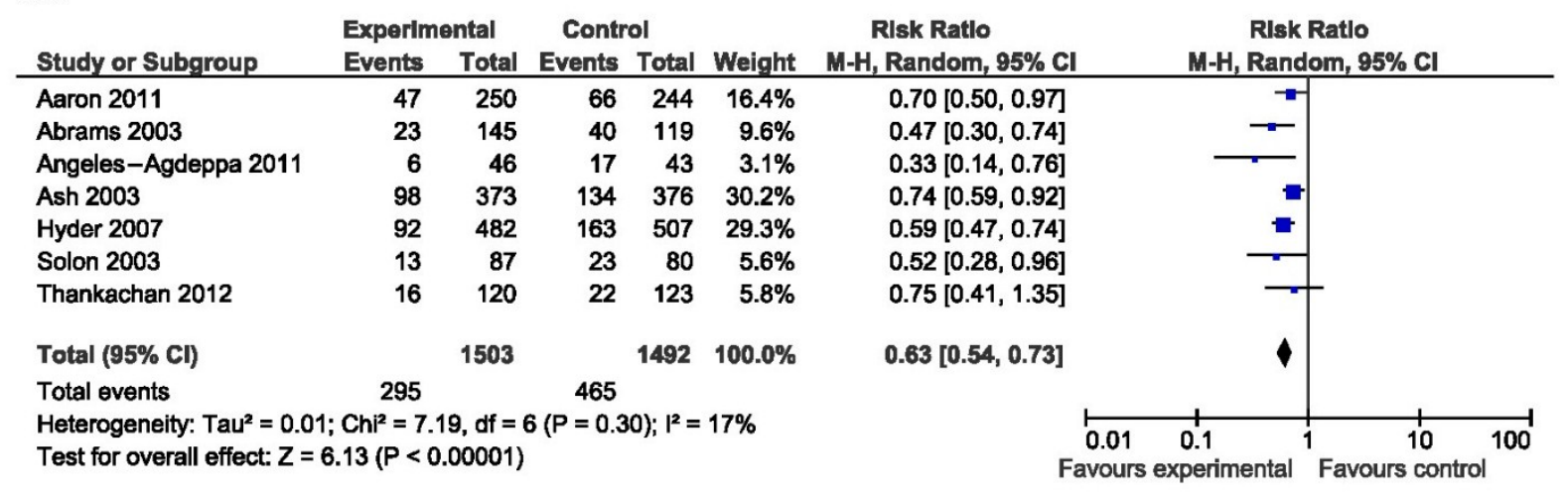

$1 \mathrm{~B}$

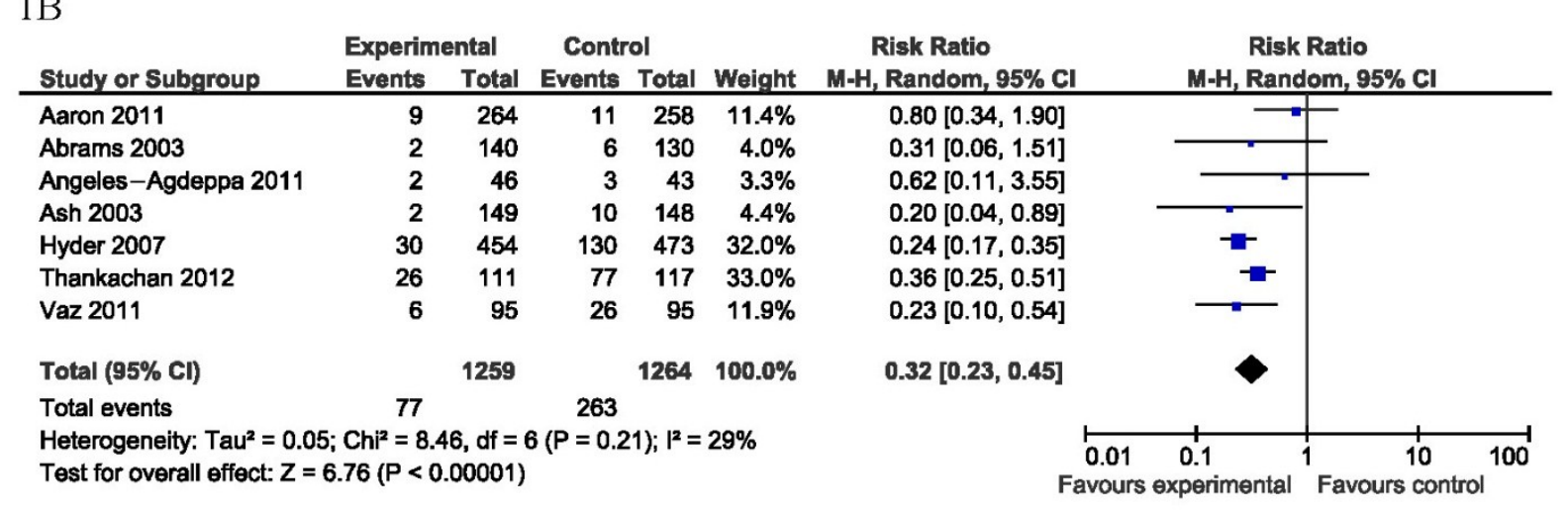

$1 \mathrm{C}$

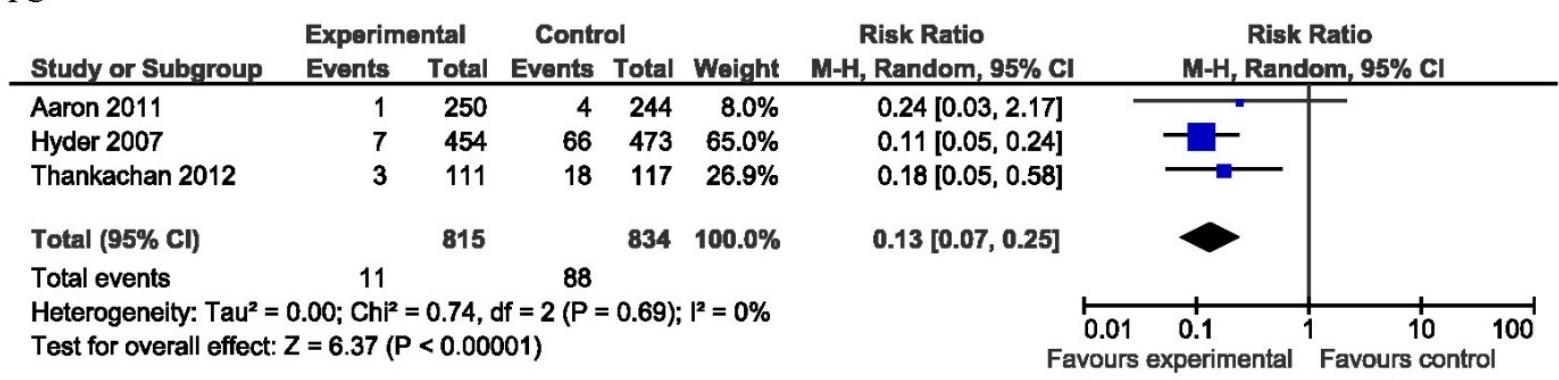

Figure 1. Forest plot of studies assessing hemoglobin and iron outcomes. Consumption of MMN beverages fortified compared to iso-caloric controls significantly reduced the risk of anemia (1A); iron deficiency (1B); and iron deficiency anemia (1C).

Study duration was not significantly associated with any of the biochemical or anthropometric outcomes measured. When comparing reconstituted powder to RTC beverages, there were no significant differences in any index except for ferritin, which demonstrated significantly less change in interventions with RTC beverages. However, after excluding the study by Aaron et al., differences were no longer significant.

A single placebo-controlled trial evaluated an 8-week intervention with a non-dairy MMN-fortified beverage in Tanzanian women who were 12-34 weeks gestation at baseline [21]. Only women who had not delivered by the end of the study period were included in analysis. Compared with women in the control group, there was a significant improvement in hemoglobin $(p=0.015)$ and serum ferritin 
$(p=0.009)$ but not in serum retinol in women in the intervention group. Consumption of iron/folic acid tablets did not differ significantly between the two groups.

\section{Discussion}

To the best of our knowledge, this is the first systematic review and meta-analysis to focus on nutritional outcomes related to consumption of MMN fortified beverages in the context of low-middle income countries. Among school children who consumed non-dairy MMN fortified beverages compared with controls consuming iso-caloric non-fortified placebo beverages, results from the meta-analysis showed significant improvements in hemoglobin and ferritin, but not in other biochemical indices. Consequently, consumption of fortified beverages significantly reduced the risk of anemia, iron deficiency, and iron deficiency anemia, but did not significantly alter the risk of other micronutrient deficiencies. In the meta-regression analyses, baseline status, intervention dose, study duration, and deworming were not significant predictors of differences in hemoglobin, ferritin, or weight change between groups.

A small but significant effect was found for weight gain in micronutrient vs. control groups despite beverages in both groups being iso-caloric. No effects were found on other anthropometric indices. Morbidity and functional outcomes, such as cognition and physical performance, could not be adequately assessed due to insufficient data points. Although meta-analysis was precluded by the scarcity of data, findings from a single MMN-fortified beverage trial conducted in pregnant women in Tanzania were consistent with results of the meta-analysis in school children.

Results from the present analysis are in general agreement with the findings from a previous systematic review on MMN fortification of foods or beverages in school children [22]. Eleven of the 12 studies in the previous systematic review were conducted in transitional or developing countries, and four of these overlapped with studies included in the present meta-analysis [4,7,14,15]. Although meta-analyses were not performed, the investigators reported an improvement in hemoglobin and iron status in eight of the 10 studies in which micronutrient outcomes were measured, as well as a reduction in anemia in seven studies. Beneficial effects on cognitive performance were found in two studies of longer duration (6 and 12 months).

Findings from the present study were similar to a recently published Cochrane review on the effects of micronutrient powders (MNP) among infants and children (6-23 months at the time of the intervention) in low-income countries [23]. In the Cochrane analysis, home fortification with MNP was shown to reduce the likelihood of anemia by $31 \%$ and iron deficiency by $51 \%$ compared with no intervention or placebo, but did not have an effect on growth measured by weight-for-age, length-for-age, and weight-for-length Z-scores. A single study included in the review reporting serum zinc as an outcome did not find an effect of MNP including $5 \mathrm{mg} / \mathrm{d}$ zinc for 6 months on zinc concentration [24]; retinol and vitamin B12 were not reported as outcomes.

The greater impact of MMN-fortified beverages on anemia and iron status compared with other biochemical outcomes in this as well as previous reviews [22,23] may have several explanations. Inconsistency in biochemical markers of micronutrient status precluded meta-analysis for several micronutrients that did show positive impacts in individual studies included in the current review. Among outcomes for which a meta-analysis was feasible, hemoglobin and ferritin were the most 
common nutritional indices measured, yielding more data points and higher power in the analysis. Furthermore, methodological limitations, including the validity of serum retinol as an index of status [25], poor sensitivity of plasma zinc [8], and differences in laboratory techniques used to measure circulating vitamin B12, may have obscured positive findings for other micronutrients.

Despite the stronger grade of evidence for hemoglobin and iron status outcomes in the present analysis, evidence suggests that combined MMN supplementation may have a greater effect than iron supplementation alone. A meta-analysis on the effects of micronutrients on growth in children below age 18 years found that iron alone had no significant effect but that MMN interventions improved linear and possibly ponderal growth [26]. This phenomenon is likely related to the frequent presence of MMN deficiencies in children in developing countries subjected to poor dietary quality coupled with frequent episodes of infection.

Several considerations should be made with regard to implementing beverage fortification strategies. Firstly, as with any nutrition strategy, the justification for fortifying a vehicle should be based on demonstrated micronutrient needs and health parameters of the population, and should take into account issues such as safe administration of micronutrient interventions [6]. In higher-income countries fortification has come under criticism due to some products on the market being fortified with micronutrients that are not necessarily deficient in the diet [27]. This practice is misleading to consumers, and may result in excessive consumption of a product due to perceptions of its health benefits. Second, there is a growing body of literature on the negative effects of sugar-sweetened beverages. While it is beyond the scope of the current research to cover this topic, considerations to limit the caloric content of beverages selected for fortification are critical due to the increasing double burdens of under- and over-nutrition in many low-middle income countries [28]. In the present meta-analysis, weight gain was modestly but significantly greater in the groups receiving MMN fortified beverages compared with controls despite the fact that the beverages were iso-caloric. This finding may suggest that specific micronutrients or their combination could have contributed to the modest weight gain observed. Most studies did not report the energy content of beverages provided, which precluded this factor being assessed in the meta-analysis. However, a single study in South Africa comparing caloric with non-caloric MMN-fortified beverages found an inverse association between sugar as a nutritive sweetener and weight-for-age $Z$-score [14].

Strengths of the present systematic review and meta-analysis include a comprehensive and targeted search strategy to identify relevant publications and a thorough quantitative analysis of available data. Limitations were the small number of beverage fortification RCT, multiple reported outcomes with insufficient data points for inclusion in meta-analysis, and incomplete information on quantities and chemical forms of micronutrients, quantities of macronutrients (particularly energy and protein), and compliance. However, despite the aforementioned limitations, results from the analyses showed a clear benefit of MMN-fortified beverage fortification on anemia and iron status, both of which are high burden global public health issues.

\section{Conclusions}

In summary, evidence from the present study suggests that the administration of non-dairy MMN-fortified beverages in the context of school settings in low-middle income countries is effective 
at improving hemoglobin and ferritin, and reducing the prevalence of anemia, iron deficiency, and iron deficiency anemia. While only one study evaluated the impacts on women, results were consistent with the broader findings in meta-analyses among children. Despite the positive impacts found in the present review, considerations to limit the caloric content of beverages selected for fortification are important if used in programs or market-based settings. Additional research is needed to investigate the effects of MMN-fortified beverages on other biochemical outcomes and population subgroups, as well as to evaluate effectiveness in programmatic settings.

\section{Acknowledgments}

Supported by The Coca Cola Company. The funding source played no role in the study design, analyses, or interpretation of results. The authors thank Steven A. Abrams (Baylor College of Medicine) and S.M. Ziauddin Hyder (World Bank) for reviewing the articles included in the review and pre-submission drafts of the manuscript; and Jan M. Peerson (University of California, Davis) for statistical consultation.

\section{Author Contributions}

All authors contributed significantly to designing and conducting the systematic review, performing the statistical analysis, analyzing data, and writing the paper. All authors have read and approved the final manuscript.

\section{Supplementary Files}

Supplemental Figure S1 - Consort flow diagram of study article selection and inclusion process. Supplemental Table S1-Micronutrient formulations for studies included in the systematic review and meta-analysis.

\section{Conflicts of Interest}

The authors declare no conflicts of interest.

\section{References}

1. Allen, L.H.; Peerson, J.M.; Olney, D.K. Provision of multiple rather than two or fewer micronutrients more effectively improves growth and other outcomes in micronutrient-deficient children and adults. J. Nutr. 2009, 139, 1022-1030.

2. Bhutta, Z.; Salam, R. Global nutrition epidemiology and trends. Annu. Nutr. Metab. 2012, 61, $19-27$.

3. Guerrant, R.L.; Lima, A.A.M.; Davidson, F. Micronutrients and infection: Interactions and implications with enteric and other infections and future priorities. J. Infect. Dis. 2000, 182, S134-S138.

4. Black, R.E.; Victora, C.G.; Walker, S.P.; Bhutta, Z.A.; Christian, P.; de Onis, M.; Ezzati, M.; Grantham-McGregor, S.; Katz, J.; Martorell, R.; et al. Maternal and child undernutrition and overweight in low-income and middle-income countries. Lancet 2013, 382, 427-451. 
5. Olney, D.K.; Rawat, R.; Ruel, M.T. Identifying potential programs and platforms to deliver multiple micronutrient interventions. J. Nutr. 2012, 142, 178S-185S.

6. Allen, L.H.; de Benoist, B.; Dary, O.; Hurrell, R. Guidelines on Food Fortification with Micronutrients; Allen, L.H., de Benoist, B., Dary, O., Hurrell, R., Eds.; World Health Organization (WHO) and Food and Agricultural Organization (FAO): Geneva, Switzerland, 2006.

7. Bhutta, Z.A.; Das, J.K.; Rizvi, A.; Gaffey, M.F.; Walker, N.; Horton, S.; Webb, P.; Lartey, A.; Black, R.E. Evidence-based interventions for improvement of maternal and child nutrition: What can be done and at what cost? Lancet 2013, 382, 452-477.

8. Brown, K.H.; Rivera, J.A.; Bhutta, Z.; Gibson, R.S.; King, J.C.; Lonnerdal, B.; Ruel, M.T.; Sandtrom, B.; Wasantwisut, E.; Hotz, C.; et al. International zinc nutrition consultative group (izincg) technical document \#1. Assessment of the risk of zinc deficiency in populations and options for its control. Food Nutr. Bull. 2004, 25, S99-S203.

9. Horton, S.; Mannar, V.; Wesley, A. Food Fortification with Iron and Iodine. Copengagen Consensus Best Practice Paper 2008. Available online: http://www.Copenhagenconsensus.Com/ sites/default/files/bpp_fortification.Pdf (accessed on 25 February 2015).

10. Angeles-Agdeppa, I.; Magsadia, C.; Capanzana, M. Fortified juice drink improved iron and zinc status of schoolchildren. Asia Pac. J. Clin. Nutr. 2011, 20, 535-543.

11. Thankachan, P.; Selvam, S.; Surendran, D.; Chellan, S.; Pauline, M.; Abrams, S.A.; Kurpad, A.V. Efficacy of a multi micronutrient-fortified drink in improving iron and micronutrient status among schoolchildren with low iron stores in india: A randomised, double-masked placebo-controlled trial. Eur. J. Clin. Nutr. 2013, 67, 36-41.

12. Yang, Z.; Huffman, S.L. Review of fortified food and beverage products for pregnant and lactating women and their impact on nutritional status. Mater. Child Nutr. 2011, 7, 19-43.

13. Vaz, M.; Pauline, M.; Unni, U.S.; Parikh, P.; Thomas, T.; Bharathi, A.V.; Avadhany, S.; Muthayya, S.; Mehra, R.; Kurpad, A.V. Micronutrient supplementation improves physical performance measures in asian indian school-age children. J. Nutr. 2011, 141, 2017-2023.

14. Taljaard, C.; Covic, N.M.; van Graan, A.E.; Kruger, H.S.; Smuts, C.M.; Baumgartner, J.; Kvalsvig, J.D.; Wright, H.H.; van Stuijvenberg, M.E.; Jerling, J.C. Effects of a multimicronutrient-fortified beverage, with and without sugar, on growth and cognition in south african schoolchildren: A randomised, double-blind, controlled intervention. Br. J. Nutr. 2013, 110, 2271-2284.

15. Hyder, S.M.; Haseen, F.; Khan, M.; Schaetzel, T.; Jalal, C.S.; Rahman, M.; Lonnerdal, B.; Mannar, V.; Mehansho, H. A multiple-micronutrient-fortified beverage affects hemoglobin, iron, and vitamin a status and growth in adolescent girls in rural bangladesh. J. Nutr. 2007, 137, 2147-2153.

16. Higgins, J.; Thompson, S.; Deeks, J.J.; Altman, D.G. Measuring inconsistency in meta-analyses. Br. Med. J. 2003, 327, 557-560.

17. Aaron, G.J.; Kariger, P.; Aliyu, R.; Flach, M.; Iya, D.; Obadiah, M.; Baker, S.K. A multi-micronutrient beverage enhances the vitamin a and zinc status of nigerian primary schoolchildren. J. Nutr. 2011, 141, 1565-1572. 
18. Abrams, S.A.; Mushi, A.; Hilmers, D.C.; Griffin, I.J.; Davila, P.; Allen, L. A multinutrientfortified beverage enhances the nutritional status of children in botswana. J. Nutr. 2003, 133, 1834-1840.

19. Ash, D.M.; Tatala, S.R.; Frongillo, E.A., Jr.; Ndossi, G.D.; Latham, M.C. Randomized efficacy trial of a micronutrient-fortified beverage in primary school children in tanzania. Am. J. Clin. Nutr. 2003, 77, 891-898.

20. Solon, F.S.; Sarol, J.N., Jr.; Bernardo, A.B.; Solon, J.A.; Mehansho, H.; Sanchez-Fermin, L.E.; Wambangco, L.S.; Juhlin, K.D. Effect of a multiple-micronutrient-fortified fruit powder beverage on the nutrition status, physical fitness, and cognitive performance of schoolchildren in the philippines. Food Nutr. Bull. 2003, 24, 129-140.

21. Makola, D.; Ash, D.M.; Tatala, S.R.; Latham, M.C.; Ndossi, G.; Mehansho, H. A micronutrient-fortified beverage prevents iron deficiency, reduces anemia and improves the hemoglobin concentration of pregnant tanzanian women. J. Nutr. 2003, 133, 1339-1346.

22. Best, C.; Neufingerl, N.; Del Rosso, J.M.; Transler, C.; van den Briel, T.; Osendarp, S. Can multi-micronutrient food fortification improve the micronutrient status, growth, health, and cognition of schoolchildren? A systematic review. Nutr. Rev. 2011, 69, 186-204.

23. De-Regil, L.M.; Suchdev, P.S.; Vist, G.E.; Walleser, S.; Pena-Rosas, J.P. Home fortification of foods with multiple micronutrient powders for health and nutrition in children under two years of age (review). Evid. Based Child Health 2013, 8, 112-201.

24. Adu-Afarwuah, S.; Lartey, A.; Brown, K.H.; Zlotkin, S.; Briend, A.; Dewey, K.G. Randomized comparison of 3 types of micronutrient supplements for home fortification of complementary foods in ghana: Effects on growth and motor development. Am. J. Clin. Nutr. 2007, 86, 412-420.

25. WHO. Serum Retinol Concentrations for Determining the Prevalence of Vitamin a Deficiency in Populations 2011. Available online: http://www.Who.Int/vmnis/indicators/retinol.Pdf (accessed on 25 February 2015).

26. Ramakrishnan, U.; Neufeld, L.M.; Gonzalez-Cossio, T.; Villalpando, S.; Garcia-Guerra, A.; Rivera, J.; Martorell, R. Multiple micronutrient supplements during pregnancy do not reduce anemia or improve iron status compared to iron-only supplements in semirural mexico. J. nutr. 2004, 134, 898-903.

27. Popkin, B.M.; Armstrong, L.E.; Bray, G.M.; Caballero, B.; Frei, B.; Willett, W.C. A new proposed guidance system for beverage consumption in the united states. Am. J. Clin. Nutr. 2006, $83,529-542$.

28. De Benoist, B.; MacLean, E.; Egli, I.; Cogswell, M. Worldwide Prevalence of Anaemia 19932005: Who Global Database of Anaemia; World Health Organization: Geneva, Switzerland, 2008.

(C) 2015 by the authors; licensee MDPI, Basel, Switzerland. This article is an open access article distributed under the terms and conditions of the Creative Commons Attribution license (http://creativecommons.org/licenses/by/4.0/). 